\title{
Impact of COVID-19 Lockdown on Glycaemic Control and Lifestyle Changes in Children and Adolescents with Type 1 And Type 2 Diabetes Mellitus
}

Hooi Peng Cheng ( $\nabla$ hooipeng100@yahoo.com )

Hospital Putrajaya https://orcid.org/0000-0002-6477-0798

Jeanne Sze Lyn Wong

Putrajaya Hospital: Hospital Putrajaya

Nalini M Selveindran

Putrajaya Hospital: Hospital Putrajaya Janet Yeow Hua Hong

Putrajaya Hospital: Hospital Putrajaya

\section{Research Article}

Keywords: COVID-19 pandemic, lockdown, type 1 diabetes mellitus, type 2 diabetes mellitus, glycaemic control, children and adolescents

Posted Date: April 27th, 2021

DOI: https://doi.org/10.21203/rs.3.rs-432636/v1

License: (9) This work is licensed under a Creative Commons Attribution 4.0 International License. Read Full License

Version of Record: A version of this preprint was published at Endocrine on July 9th, 2021. See the published version at https://doi.org/10.1007/s12020-021-02810-1. 


\section{Abstract}

Aims: Malaysia implemented nation-wide lockdown from $18^{\text {th }}$ March till $5^{\text {th }}$ May 2020 to mitigate the spread of coronavirus disease (COVID-19). This study aimed to examine the impact of the lockdown on glycaemic control and lifestyle changes in children and adolescents with type 1 (T1DM) and 2 diabetes mellitus (T2DM) aged less than 18 years old.

Methods: In this cross-sectional study, interviews and a standardised questionnaire comparing lifestyle changes before and during the lockdown were performed in follow-up clinic visits after the lockdown. Anthropometry measurements and glycated haemoglobin ( $\mathrm{HbA} 1 \mathrm{c})$ values were compared three months prior and after the lockdown.

Results: Participants were 93 patients with T1DM (4.64 \pm 3.10 years) and 30 patients with T2DM (11.08 \pm 3.47 years). Male gender, T2DM and pubertal adolescents were found to have a significant deterioration in glycaemic control. A significant increment of HbA1c was observed in patients with T2DM $(8.5 \pm 0.40 \%$ vs $9.9 \pm 0.46 \%)$, but not in patients with T1DM ( $8.6 \pm 0.28 \%$ vs $8.7 \pm 0.33 \%)$. Contrarily, there was an improved glycaemic control in pre-pubertal T1DM children likely due to parental supervision during home confinement. Weight and BMI SDS increased in T1DM patients but surprisingly reduced in T2DM patients possibly due to worsening diabetes control. Reduced meal frequency mainly due to skipping breakfast, reduced physical activity level scores, increased screen time and sleep duration were observed in both groups.

Conclusions: Adverse impact on glycaemic control and lifestyle were seen mostly in patients with T2DM and pubertal adolescent boys.

\section{Background}

The catastrophic outbreak of novel severe acute respiratory syndrome coronavirus 2 (SARS-CoV-2) in Wuhan City, China in December 2019, has spread globally within months and was declared a pandemic since March 2020 by the World Health Organization. In Malaysia, the first case of COVID-19 was reported on 25th January 2020. At the time of writing in April 2021, Malaysia has recorded a total of 365829 cases with 1353 deaths.

In the effort to increase social distancing and to mitigate the spread of COVID-19, a nationwide lockdown known as the Movement Control Order (MCO) was implemented in Malaysia from 18th March until 3rd May 2020. Schools were closed and all non-essential businesses, outdoor sports, leisure activities, travel between states and districts were restricted.

The paediatric diabetes service in Putrajaya Hospital is a tertiary service which caters for referrals from states all throughout Malaysia. The implementation of the MCO resulted in difficulties in access to care for many patients with diabetes. This was particularly apparent in families who were from the lower socio-economic group. In an attempt to reduce physical congestions at the clinics, alternative means of 
communications with patients and families were implemented such as phone consultations and emails. Patients who were from different states or districts were also co-managed at the nearest major hospitals from them. Most studies assessing impact of COVID-19 lockdown involved adult patients with T1DM with only a few studies involving children and adolescents. Results of these studies were conflicting. Improvement of glycaemic control were observed in Spanish adults with T1DM [1, 2], Italian children and adolescents with T1DM [3, 4] and Indian adults with T2DM [5]. T1DM children and adolescents in India [6] and Saudi Arabia [7], T1DM Japanese adults [8], and T2DM Turkish adults [9] were reported to have worsening of glycaemic control. However, generally, glycaemic control was reported to be unchanged in most children and youths with T1DM [10-13]. To our knowledge, there was no reported study on the impact of COVID-19 lockdown in adolescents with T2DM. We hypothesized that the MCO and the COVID19 pandemic would have a negative impact in the management of young people with diabetes.

The aim of this study was to examine the impact of the lockdown caused by COVID-19 pandemic on glycemic control, body mass index (BMI) and lifestyle changes in children and adolescents with type 1 and 2 diabetes mellitus.

\section{Methods}

\section{Patients and Study Design}

This was a cross-sectional interview and questionnaire-based study conducted in our centre from June until December 2020. Inclusion criteria were all patients diagnosed with T1DM and T2DM below the age of 18 years who had been under our centre follow-up for at least 6 months prior to the lockdown and had at least one physical clinic follow-up after the lockdown. During the lockdown period (18th March till 5th May 2020), phone consultations had been carried out for most patients to monitor their general wellbeing, lifestyle changes, self-monitoring of blood glucose, and compliance to insulin and medications.

After the lockdown was lifted, all participants and their parents had a follow-up visit to the paediatric endocrine clinic within the study duration. At the clinic, they were interviewed about their diabetes management and lifestyle changes before and during the lockdown by the same investigator for consistency and to eliminate bias. Participants were also required to fill-up a standardised questionnaire on their lifestyle changes which include dietary habits, screen time, sleep duration and physical activities before and during the lockdown. For young patients aged 9 years and below, the questionnaires were filled by the parents. For adolescents 10-18 years old, questionnaires were self-administered with assistance from the parents as required. Level of physical activities were assessed using the Physical Activity Questionnaire for Children (PAQ-C) and the Physical Activity Questionnaire for Older Children (PAQ-A) adapted from Crocker et al. [14] and Kowalski et al. [15]. Baseline clinical data and laboratory test results were retrieved from the patients' electronic medical records.

\section{Anthropometry}


Anthropometric measurements (height, weight, BMI) were retrieved from the electronic medical records (EMR) during the last outpatient visit prior to COVID-19 lockdown. After the lockdown was lifted, anthropometric parameters were measured when patients returned to outpatient visits. Body mass index (BMI) was calculated from weight and height $\left(\mathrm{kg} / \mathrm{m}^{2}\right)$. All the anthropometric measurements was converted to standard deviation (SD) score according to age and gender using WHO growth charts. Tanner staging was utilised to assess pubertal status.

\section{Glycaemic control}

Glycaemic control was determined by glycated haemoglobin (HbA1c), which provide the average plasma glucose concentration within the past 3 months. Results of HbA1c were obtained from EMR of the last clinic visit prior to COVID-19 lockdown. Repeated HbA1c measurement was made during follow-up visits to the paediatric endocrine clinic post-lockdown.

\section{Physical activity}

Physical activity is defined as any body movement produced by skeletal muscle that requires energy expenditure. The Physical Activity Questionnaire for Children (PAQ-C) and the Physical Activity Questionnaire for Older Children (PAQ-C) were adapted from Crocker et al. [14] and Kowalski et al. [15] to assess the general level of physical activity of the children and adolescents. A standardized questionnaire with translation into the local Malay Language and adaption of the type of physical activity into the local context [16] was utilised. The PAQ-C consists of 9 items and PAQ-A consisted of 8 items; each scored on a 5-point scale. Both are self-administered with a 7 days recall tools to evaluate moderate to vigorous physical activity for school-going children and adolescents. Physical activity composite score was calculated by the mean of all the items. The score ranges from 1 to 5 , a higher score indicated higher levels of physical activity. Participants 6 to 13 years old would be required to answer the Physical Activity Questionnaire for Children (PAQ-C), while adolescents age 14 to 18 years would be required to answer the Physical Activity Questionnaire for Older Children (PAQ-A). Questionnaires are self-administered by the participants with assistance from the parents if required. However, for participants aged 6 to 9 years old, parents are required to complete the questionnaire. Each participant or parent would be required to answer 2 sets of Physical Activity Questionnaire to reflect the physical activity prior to and during lockdown periods.

\section{Statistical analysis}

Data analyses were performed using SPSS 23.0 statistical analysis software (SPSS Inc., Chicago, IL, USA). Continuous variables were presented as mean \pm standard deviation if normally distributed. Categorical variables were shown as the number and percentage of patients involved. Repeated measures ANOVA were used to compare differences between the two phrases. Statistical significance was defined as two-tailed $p$-value $<0.05$.

\section{Results}




\section{Demographic and clinical characteristics}

A total of 123 patients were recruited into the study. Ninety three $(75 \%)$ were patients with T1DM and the remainder 30 (25\%) T2DM. The mean age for participants with T1DM was $11.08 \pm 3.47$ years, mean duration of diabetes $4.64 \pm 3.10$ years and mean age of diagnosis $6.43 \pm 3.31$ years. All participants with T1DM was on multiple daily insulin injections (basal-bolus regimen), except for six patients (6.5\%) who were on continuous subcutaneous infusion insulin pump therapy. Two patients $(2.15 \%)$ have diabetic hepatic glycogenopathy and diabetic nephropathy.

For T2DM patients, the mean age was $13.81 \pm 2.03$ years (9.9-18 years), mean duration of diabetes 2.61 \pm 1.78 years and mean age of diagnosis $11.15 \pm 1.46$ years. All participants with T2DM were on metformin. Eighteen patients were also on basal insulin. Four patients (13.33\%) had diabetic nephropathy. As expected, T2DM participants had higher BMI compared to T1DM and they were also older. None of our patients developed diabetic ketoacidosis or Covid-19 infection during the study period. Demographic and clinical characteristics of the participants are summarised in Table 1. 
Table 1

Anthropometric, biochemical and lifestyle characteristic at baseline

\begin{tabular}{|c|c|c|c|}
\hline & T1DM & T2DM & p-value \\
\hline \multicolumn{4}{|l|}{ Demographic } \\
\hline Total Number of patients & 93 & 30 & \\
\hline Age (years) & $11.08(3.47)$ & $13.81(2.03)$ & $<0.005$ \\
\hline Gender & $44(47.3)$ & $10(33.3)$ & 0.180 \\
\hline - Male, n (\%) & $49(52.7)$ & $20(66.7)$ & \\
\hline \multicolumn{4}{|l|}{ - Female, n (\%) } \\
\hline Weight SDS & $-0.66(1.23)$ & $1.69(1.02)$ & $<0.001$ \\
\hline Height SDS & $-0.78(1.17)$ & $-0.26(1.25)$ & 0.041 \\
\hline BMI SDS & $-0.29(1.13)$ & $1.86(0.60)$ & $<0.005$ \\
\hline \multicolumn{4}{|l|}{ Clinical characteristics } \\
\hline Age of diagnosis (years) & $6.43(3.31)$ & $11.15(1.46)$ & $<0.001$ \\
\hline Diabetes duration (years) & $4.64(3.10)$ & $2.61(1.78)$ & $<0.005$ \\
\hline Diabetic complication & $0(0)$ & $0(0)$ & $0.063^{*}$ \\
\hline - Diabetic retinopathy, n (\%) & $2(2.15)$ & $4(13.33)$ & \\
\hline - Diabetic nephropathy, n (\%) & $0(0)$ & $0(0)$ & \\
\hline - Diabetic neuropathy, n (\%) & $2(2.15)$ & $0(0)$ & \\
\hline - Other, n (\%) & $2(2.15)$ & $0(0)$ & \\
\hline \multicolumn{4}{|l|}{ - > 1 complication, n (\%) } \\
\hline Mean insulin dose (unit/kg/day) & $0.97(0.23)$ & $0.39(0.38)$ & $<0.001$ \\
\hline On multiple daily injection therapy, $\mathrm{n}(\%)$ & $87(93.5)$ & $18(60.0)$ & \\
\hline Insulin pump user, n (\%) & $6(6.5)$ & $0(0)$ & $0.587^{*}$ \\
\hline History of severe hypoglycaemia, n (\%) & $5(5.4)$ & $1(3.33)$ & $1.000 *$ \\
\hline Glycated haemoglobin (\%) & $9.5(2.24)$ & $9.0(2.54)$ & 0.247 \\
\hline Receiving medical advice by telephone, $\mathrm{n}(\%)$ & $55(59.1)$ & $1(3.2)$ & $<0.001^{*}$ \\
\hline \multicolumn{4}{|l|}{ Lifestyle parameters } \\
\hline Meal frequency & $4.86(0.96)$ & $4.10(1.03)$ & $<0.005$ \\
\hline PAQ score & $2.03(0.56)$ & $1.83(0.47)$ & 0.096 \\
\hline
\end{tabular}




\begin{tabular}{|llll|}
\hline & T1DM & T2DM & p-value \\
\hline Screen time (hour) & $2.05(1.76)$ & $2.22(1.67)$ & 0.647 \\
\hline Sleeping duration (hour) & $8.14(1.26)$ & $7.65(0.98)$ & 0.055 \\
\hline
\end{tabular}

Data were expressed as mean with standard deviation in parenthesis. Continuous variables were investigated by independent $\mathrm{T}$ test, and categorical variables were analysed by Chi Square test (*Fisher exact test for non-parametric distribution).

SDS, SD score; BMI, body mass index; PAQ, Physical Activity Questionnaire.

\section{Effects on glycaemic control}

Comparison of glycaemic control (represented by HbA1c level) according to various factors before and after MCO are summarised in Table 2. Significant deteriorations were observed in male patients, those with T2DM and pubertal adolescents. In contrast, $\mathrm{HbA} 1 \mathrm{c}$ of younger pre-pubertal patients improved after the lock down ( $p$ 0.006). There was no overall significant changes in glycaemic control in T1DM patients. 
Table 2

Factors influencing glycaemic control before and after the lockdown

\begin{tabular}{|lllll|}
\hline & \multicolumn{2}{l}{ HbA1c } & Mean diff & p-value \\
\cline { 2 - 3 } & Before the lockdown & After the lockdown & \\
\hline Overall & $8.5(0.26)$ & $9.3(0.30)$ & 0.8 & 0.001 \\
Gender & $8.5(0.34)$ & $9.8(0.40)$ & 1.3 & 0.015 \\
- Male & $8.5(0.28)$ & $8.8(0.32)$ & 0.3 & \\
- Female & & & & \\
\hline Type of diabetes & $8.6(0.28)$ & $8.7(0.33)$ & 0.1 & 0.012 \\
- T1DM & $8.5(0.40)$ & $9.9(0.46)$ & 1.4 & \\
- T2DM & & & & \\
\hline Baseline HbA1c & $6.7(0.37)$ & $7.9(0.42)$ & 1.2 & 0.051 \\
- Less than 7.5\% & $10.4(0.28)$ & $10.7(0.33)$ & 0.3 & \\
- $\geq 7.5 \%$ & & & & \\
\hline Puberty & $8.2(0.46)$ & $7.5(0.53)$ & -0.7 & 0.006 \\
- Pre-pubertal & $8.3(0.28)$ & $9.7(0.32)$ & 1.4 & \\
- In puberty & $9.1(0.65)$ & $10.6(0.75)$ & 1.5 & \\
- Completed puberty & & & & \\
\hline
\end{tabular}

Data were expressed as adjusted mean with standard deviation in parenthesis. Continuous variables were analysed by One-way Repeated Measures ANOVA test using General Linear Model, after adjusted by age $(p$-value $=0.001)$ and diabetes duration $(p$-value $=0.072)$.

\section{Lifestyle changes during lockdown}

There were significant changes related to lifestyle in both T1DM and T2DM patients during the lockdown. There was a significant increase in BMI and weight (from $34.58 \pm 14.29 \mathrm{~kg}$ to $37.43 \pm 14.57 \mathrm{~kg}$ post lockdown) in T1DM patients ( $p$ 0.000). However, there was a reduction in weight and BMI in T2DM patients. Meal frequency was reduced in both groups, mainly due to breakfast skipping. Pre-existing low physical activity level in both groups deteriorated further during the lockdown. There was also a significant increment in screen time of 1.9-2.9 folds in T2DM and T1DM groups. Sleep duration had also increased in both groups. Comparison of lifestyle changes before and during the lockdown in T1DM and T2DM patients are summarised in Tables 3 and 4 respectively. 
Table 3

Clinical and lifestyle characteristics before and during the MCO in T1DM patients

\begin{tabular}{|lllllll|}
\hline & Male & \multicolumn{5}{c|}{ Female } \\
\hline & $\begin{array}{l}\text { Before } \\
\text { lockdown }\end{array}$ & $\begin{array}{l}\text { During } \\
\text { lockdown }\end{array}$ & $\begin{array}{l}\text { p- } \\
\text { value }\end{array}$ & $\begin{array}{l}\text { Before } \\
\text { lockdown }\end{array}$ & $\begin{array}{l}\text { During } \\
\text { lockdown }\end{array}$ & $\begin{array}{l}\text { p- } \\
\text { value }\end{array}$ \\
\hline Weight SDS & $-1.00(1.28)$ & $-0.91(1.26)$ & 0.000 & $-0.43(1.10)$ & $-0.27(1.06)$ & 0.000 \\
\hline BMI SDS & $-0.50(1.17)$ & $-0.42(1.08)$ & 0.000 & $-0.18(1.08)$ & $-0.03(0.97)$ & 0.000 \\
\hline Meal frequency & $4.86(0.98)$ & $4.48(1.05)$ & 0.000 & $4.85(0.97)$ & $4.60(0.94)$ & 0.323 \\
\hline PAQ score & $2.08(0.70)$ & $1.77(0.55)$ & 0.000 & $2.00(0.38)$ & $1.89(0.47)$ & 0.000 \\
\hline $\begin{array}{l}\text { Screen time } \\
\text { (hours) }\end{array}$ & $2.27(1.92)$ & $5.63(2.50)$ & 0.001 & $1.84(1.63)$ & $5.25(2.79)$ & 0.000 \\
\hline $\begin{array}{l}\text { Sleep duration } \\
\text { (hours) }\end{array}$ & $8.07(1.21)$ & $9.06(1.39)$ & 0.033 & $8.18(1.31)$ & $9.33(1.33)$ & 0.000 \\
\hline
\end{tabular}

Data were expressed as mean with standard deviation in parenthesis. Continuous variables were analysed by paired sample $T$ test.

SDS, SD score; BMI, body mass index.

Table 4

Clinical and lifestyle characteristics before and during the MCO in T2DM patients

\begin{tabular}{|lllllll|}
\hline & Male & \multicolumn{5}{c|}{ Female } \\
\hline & $\begin{array}{l}\text { Before } \\
\text { lockdown }\end{array}$ & $\begin{array}{l}\text { During } \\
\text { lockdown }\end{array}$ & $\begin{array}{l}\text { p- } \\
\text { value }\end{array}$ & $\begin{array}{l}\text { Before } \\
\text { lockdown }\end{array}$ & $\begin{array}{l}\text { During } \\
\text { lockdown }\end{array}$ & $\begin{array}{l}\text { p- } \\
\text { value }\end{array}$ \\
\hline Weight SDS & $2.32(1.10)$ & $2.08(1.11)$ & 0.003 & $1.40(0.85)$ & $1.27(0.89)$ & 0.027 \\
\hline BMI SDS & $2.12(0.65)$ & $1.98(0.74)$ & 0.038 & $1.69(0.58)$ & $1.60(0.62)$ & 0.080 \\
\hline Meal frequency & $3.90(1.10)$ & $3.40(0.84)$ & 0.096 & $4.24(1.00)$ & $3.67(0.91)$ & 0.055 \\
\hline PAQ score & $1.91(0.56)$ & $1.59(0.41)$ & 0.066 & $1.79(0.41)$ & $1.62(0.40)$ & 0.063 \\
\hline $\begin{array}{l}\text { Screen time } \\
\text { (hours) }\end{array}$ & $3.05(1.71)$ & $5.90(3.25)$ & 0.001 & $1.81(1.50)$ & $5.21(3.57)$ & 0.000 \\
\hline $\begin{array}{l}\text { Sleep duration } \\
\text { (hours) }\end{array}$ & $7.80(0.71)$ & $9.65(0.94)$ & 0.001 & $7.64(1.12)$ & $9.55(1.67)$ & 0.000 \\
\hline
\end{tabular}

Data were expressed as mean with standard deviation in parenthesis. Continuous variables were analysed by paired sample $T$ test.

SDS, SD score; BMI, body mass index. 


\section{Discussion}

To the best of our knowledge, this was the first study comparing the impact of COVID-19 lockdown towards glycaemic control and lifestyle changes in children and adolescents with T1DM and T2DM. There was no similar study conducted in adolescents with T2DM to date. We reported overall deterioration in glycaemic control which was more apparent in patients with T2DM, male gender and pubertal adolescents. This cross sectional study also demonstrated marked lifestyle changes which occurred during the lockdown.

As illustrated in Table 2, T2DM patients, male gender and pubertal adolescents were the affected groups in term of the glycaemic control. In contrast, glycemic control in the younger pre-pubertal children with T1DM had significantly improved post-lockdown. This was likely due to more parental supervision of insulin injections and overall diabetes care in this young group of patients. Barbara Predieri et al [4] reported an overall improved glycemic control in Italian children and adolescents $(n=62$, mean age 11.1 +/- 4.37 years) with T1DM using real-time continuous glucose monitoring (CGM) during lockdown with better improvement seen in the pre-pubertal children. They hypothesized that the improvement could result from more regular scheduled mealtimes without higher food intake and more attention on diabetes care by parents who were "forced" to stay at home. These findings in the pre-pubertal T1DM children were similar to ours. However, we did not observed an overall glycaemic improvement in our T1DM participants likely because of the different cohort of patients and methods of assessment of glycemic control. The Italian cohort consisted of patients who already had a relatively good baseline glycemic control and with sensor CGM use prior to inclusion of the study. They also had access to telemedicine during the lockdown. Hence the results may not be generalizable to patients with poorer control or who do not have access to technology. The vast majority of our patients are on self-monitoring blood glucose by finger-pricks. Only one patient who was on insulin pump therapy was using CGM. The usage of CGM and flash glucose monitoring is limited here due to cost issues. Most of our T1DM patients had virtual consultations in the form of phone calls from the paediatric endocrine team during the lockdown. Access to telemedicine was limited in our local setting due various factors which include a lack of human resources, a suitable app application and the lack to technology access by some patients. While there was no overall improvement, there was also no significant difference in the HbA1c of our T1DM patients before and post-lockdown (8.6\% vs $8.7 \%$ ) when analysed separately from other confounding factors. This was similar to the study by Wu XM et al [10] who reported no deterioration in glycaemic control in their cohort of Chinese children and teenagers $(n=43)$ using CGM.

In contrast to T1DM patients whom glycaemic control were not affected by the lockdown, there was a significant deterioration in $\mathrm{HbA} 1 \mathrm{c}$ (8.5\% pre-lockdown vs $9.9 \%$ post-lockdown) in T2DM patients. We hypothesized the reasons for this could be multifactorial. Firstly, lifestyle management is a cornerstone in T2DM which is related to excess weight and insulin resistance. While having a healthy lifestyle is also important for T1DM patients, the mainstay of treatment for T1DM is insulin. During the lockdown, there had been a reduction in physical activity levels and increment in screen time. Interestingly despite this, there was a reduction in the weight and BMI SDS of T2DM patients compared to T1DM patients who had 
gained weight. It is likely the significant worsening of glycemic control in T2DM patients with a mean $\mathrm{HbA} 1 \mathrm{c}$ of $9.9 \%$ post lockdown, resulted in a catabolic state and weight loss.

The lockdown has had a worse glycemic effect on pubertal adolescents. Adolescence and teenage years are filled with physical, hormonal and emotional changes. There is also a shift in independence from the parents during this period and more reliance on peers support. All these may make adolescents more susceptible to major changes in routines during the lockdown resulting in depression, anxiety, boredom and a lack of motivation in diabetes management. In addition, most adolescents are already selfmanaging their diabetes and self-administering their insulin or medications and are less receptive to perceived adults' interference. Hence, during the home confinement, it was likely there was not much extra benefit in terms of parental supervision for the adolescents as compared to the younger children. This was a reminder to us that our T2DM patients require equal or even more medical attention as compared to T1DM patients during this pandemic. Continued encouragement to maintain good diabetes self-care and access to professional mental health professionals for all patients and their families should also be provided if needed.

Interestingly, the glycaemic control of our male patients were more adversely affected during this unprecedented time. We also observed that there was more changes in lifestyle in almost all aspects in boys compared to girls. This was not in keeping with previous reports that female gender was a risk factor for poorer glycaemic control $[17,18]$ attributed to greater psychological affect [19]. Possible reasons for our findings are that boys tend to be more physically active and do not cope so well with prolonged periods indoors.

Both groups had exhibited marked sedentary lifestyle during the confinement at home. According to World Health Organization [20] and Malaysian Dietary Guidelines for Children and Adolescents [21], children and adolescents should practise at least 60 minutes of moderate or vigorous intensity physical activity daily. Unfortunately, the physical activity level of our patients were already low prior to lockdown and further reduced during the lockdown. Physical activity was found to be reduced from 540 minutes per week before COVID-10 pandemic to 105 minutes per week during the pandemic among children and adolescents aged 6-17 years old in Shanghai [22]. For our T1DM patients, time spent for physical activities decreased from 226 minutes per week before to 213 minutes per week during the lockdown. The decline was more marked in our T2DM patients, in which it dropped from 229 minutes per week to 187 minutes per week. An Italian study involving a small cohort of 13 T1DM patients reported that glycaemic control improved in those who maintained physical activity during home confinement [23]. While there is restriction in outdoor activities, innovative ways to maintain physical activity levels including appropriate indoor exercises should be encourage. For the adolescents and teenagers, following popular and credible online physical trainers who share various indoor exercise videos on channels such as YouTube could be beneficial. Short e.g. 10 minutes but multiple exercise sessions a day may also be more practical in an indoor setting. Different types of physical activities indoors and its impact on glycaemic control would need further evaluation. 
Screen time which includes exposure to television, computers and hand phones in our patients had increased exponentially during the lockdown. This was expected with schools closure, introduction to online classrooms, boredom and restrictions of outdoor activities. During the lockdown period, only 6 of our patients (5.9\%) fulfilled the recommendation by American Academy of Pediatrics [24] to limit daily screen time to less than 2 hours compared to 55 patients (44.7\%) pre-lockdown. In Norway, increment in $\mathrm{HbA} 1 \mathrm{c}$ was reported with every hour of watching television in their population-based study involving 538 children and adolescents with type 1 diabetes [25].

During the lockdown, there was modification of sleep wake rhythm among some of our children and adolescents. The American Academy of Sleep Medicine recommends that toddlers (aged 3-5 years) should obtain 10-13 hours of sleep, school-going children (ages 6-12 years) 9-12 hours and adolescents 8-10 hours [26]. In our study, 70 (72.9\%) T1DM patients and 26 (83.9\%) T2DM patients had adequate sleep during the lockdown period. Most patients had increased sleep in the morning due to school closure. A shorter sleep duration was associated with poor glycaemic control. Jaser et al. [27] found that T1DM children who slept more than 9 hours per night had lower HbA1c (7.8\%) compared to those who slept less than 9 hours per night $(8 \%)(p=0.02)$. However, we did not analysed this in our study. Our patients also had less meal frequency during the lockdown. $12 \%$ of participants skipped their breakfast attributed to waking up later in the morning with school closure.

Study limitations

There were a few limitations in our study. Firstly, assessment of glycaemic control was only based on $\mathrm{HbA1c}$. Virtually all our patients were self-monitoring their home blood glucose by finger pricks with only patient using a CGM sensor. We were not able to analyse the home blood glucose levels of patients due to infrequent monitoring at home, lack of documentations in the glucose diary and forgetting to bring their glucometer on clinic follow-up. The poor compliance in monitoring blood glucose levels were likely to be multifactorial including lack of motivation, poor compliance, needle phobia and significant cost of glucose strips and needles. The other limitation was recall bias could exist as the lifestyle changes were recalled by patients and parents only after the lockdown has ended. The sample size of our T2DM participants was also relatively small.

Study strengths

The strength of our study was that the interviews were conducted by the same investigator to limit bias with the help of a standardised questionnaire which was filled up by the patients or parents. The overall sample size of our cohort was also larger than reported in previous studies involving children and adolescents with T1DM. Adequate and accurate clinical data of the participants were also readily available from our electronic medical records.

\section{Conclusion}


This study demonstrated that the lockdown resulting from COVID-19 pandemic had resulted in adverse glycaemic effects particularly affecting patients with T2DM and pubertal boys. There was also a negative lifestyle change with increased screen time, reduction in physical activity and deterioration in sleep quality in these young people. Our findings highlight that children and adolescents with diabetes especially type 2 and adolescent boys are vulnerable to deterioration of care and would need continued medical access for advice and support throughout this pandemic. They should also be encouraged to maintain a healthy lifestyle as much as possible during these difficult times. A follow-up study assessing emotional and psychological impact in children and adolescents with diabetes should be done.

\section{Abbreviations}

T1DM Type 1 Diabetes Mellitus

T2DM Type 2 Diabetes Mellitus

HbA1c glycated haemoglobin

BMI Body Mass Index

\section{Declarations}

\section{Funding}

This research did not receive any specific grant from funding agencies in the public, commercial, or notfor-profit sectors.

\section{Conflicts of interest/ Competing interests}

The authors have no relevant financial or non-financial interests to disclose.

\section{Authors' Contributions}

All authors contributed to the study conception and design. Material preparation, data collection and analysis were performed by Hooi Peng Cheng. The first draft of the manuscript was written by Hooi Peng Cheng and all authors commented on previous versions of the manuscript. All authors read and approved the final manuscript.

\section{Ethics Approval}

The study was conducted in compliance with ethical principles outlined in the Declaration of Helsinki and Malaysian Good Clinical Practice Guideline. The study was approved by Medical Research and Ethics Committee (MREC), Ministry of Health (NMRR-20-1678-55949). Parental written informed consent and patient assent/consent were obtained prior to data collection. 
$\underline{\text { Consent to Participate }}$

Written informed consent was obtained from the parents and patients.

\section{Consent for Publication}

Parents and patients signed informed consent regarding publishing their data.

\section{ACKNOWLEDGEMENT}

We would like to acknowledge Director General of Health Malaysian for the approval for this publication. We would also like to thank Tee ES et al. [16] for the Malay translation of PAQ-C and PAQ-A questionnaires.

\section{References}

1. Fernández E, Cortazar A, Belindo V. Impact of COVID-19 lockdown on glycemic control in patients with type 1 diabetes. Diabetes Research and Clinical Practice 166:108348(2020). https://doi.org/10.1016/j.diabres.2020.108348

2. Pla B, Arranz A, Knott C, Sampedro M, Jiménez S, Hernando I, Marazuela M. Impact of COVID-19 Lockdown on Glycemic Control in Adults with Type 1 Diabetes Mellitus. Journal of the Endocrine Society 4(12):1-8(2020)

3. Marigliano M, Maffeis C. Glycemic control of children and adolescents with type 1 diabetes improved after COVID-19 lockdown in Italy. Acta Diabetologica 2:1-4(2021). doi: 10.1007/s00592-020-01667-6.

4. Predieri B, Leo F, Candia F, Lucaccioni L, Madeo SF, Pugliese M, Vivaccia V, Bruzzi P, lughetti L. Glycemic Control Improvement in Italian Children and Adolescents With Type 1 Diabetes Followed Through Telemedicine During Lockdown Due to the COVID-19 Pandemic. Frontiers in Endocrinology 11:595735(2020). https://doi.org/10.3389/fendo.2020.595735

5. Rastigu A, Hiteshi P, Bhansali A. Improved glycemic control amongst people with long-standing diabetes during COVID-19 lockdown: a prospective, observational, nested cohort study. International Journal of Diabetes in Developing Countries 40:476-481(2020)

6. Verma A, Rajput R, Verma S, Balania VKB, Jangra B. Impact of lockdown in COVID 19 on glycemic control in patients with type 1 diabetes mellitus. Diabetes \& Metabolic Syndrome: Clinical Research \& Reviews 14 1213-1216(2020). https://doi.org/10.106/j.dsx.2020.07.016

7. Agha AAE, Alharbi RS, Almohammadi OA, Yousef SY, Sulimani AE, Alaama RA. Impact of COVID-19 lockdown on glycemic control in children and adolescents. Saudi Med J 42(1):46-48 (2021)

8. Hosomi Y, Munekawa C, Hashimoto Y, Okamura T, Takahashi F, Kawano R, Nakajima H, Majima S, Senmaru T, Nakanishi N, Ushigome E, Hamaguchi M, Yamazaki M, Fukui M. Effect of COVID-19 pandemic on the lifestyle and glycemic control in patients with type 1 diabetes: A retrospective cohort study. Research Square. https://doi.org/10.21203/rs.3.rs-83364/v1 
9. Önmez A, Gamsizkan Z, Özdemir S, Kesikbas E, Gökosmanoğlu F, Torun S, Cinemre H. The effect of COVID-19 lockdown on glycemic control in patients with type 2 diabetes mellitus in Turkey. Diabetes \& Metabolic Syndrome: Clinical Research \& Reviews 14 1963-1966(2020). https://doi.org/10.1016/j.dsx.2020.10.007

10. Wu X, Luo S, Zheng X, Ding Y, Wang S, Ling P, Yue T, Xu W, Yan J, Weng J. Glycemic control in children and teenagers with type 1 diabetes around lockdown for COVID-19: A continuous glucose monitoring-based observational study. Journal of Diabetes Investigation (2021). https://doi.org/10.1111/jdi.13519

11. Di Dalmazi G, Maltoni G, Bongiorno C, Tucci L, Natale VD, Moscatiello S, Laffi G, Pession A, Zucchini S, Pagotto U. Comparison of the effects of lockdown due to COVID-19 on glucose patterns among children, adolescents and adults with type 1 diabetes: CGM study. BMJ Open Diab Res Care 8:e001664(2020). https://doi.org/10.1136/bmjdrc-2020-001664

12. Troncone A, Chianese A, Zanfardino A, Cascella C, Piscopo A, Borriello A, Rollato S, Casaburo F, Testa $\mathrm{V}$, lafusco $\mathrm{D}$. Disordered eating behaviors in youths with type 1 diabetes during COVID-19 lockdown: an exploratory study. Journal of Eating Disorders 8:76(2020). https://doi.org/10.1186/s40337-02000353-w

13. Tornese G, Ceconi V, Monasta L, Carletti C, Faleschini E, Barbi E. Glycemic Control in Type I Diabetes Mellitus During COVID-19 Quarantine and the Role of In-Home Physical Activity. Diabetes Technology \& Therapeutics 22(6):462-7(2020). https://doi.org/10.1089/dia.2020.0169

14. Crocker PRE, Bailey DA, Faulkner RA, Kowalski KC, McGrath R. Measuring general levels of physical activity: preliminary evidence for the Physical Activity Questionnaire for Older Children. Med Sci Sports Exerc. 29:1344-9(1997)

15. Kowalski KC, Crocker PRE, Donen RM. The physical Activity Questionnaire for Older Children (PAQ-C) and Adolescents (PAQ-A) Manual. Saskatoon: College of Kinesiology, University of Saskatchewen; (2004)

16. Tee ES, Nurliyana AR, Norimah AK, Mohamed HJBJ, Tan SY, Appukutty M, et al. Breakfast consumption among Malaysian primary and secondary school children and relationship with body weight status - Findings from the MyBreakfast Study. Asia Pac J Clin Nutr 27(2):421-432 (2018)

17. Gerstl EM, Rabl W, Rosenbauer J, et al. Metabolic control as reflected by HbA1c in children, adolescents and young adults with type- 1 diabetes mellitus: combined longitudinal analysis including 27,035 patients from 207 centers in Germany and Austria during the last decade. Eur $\mathrm{J}$ Pediatr 167(4):447-53(2008)

18. Setoodeh A, Mostafavi F, Rabbani A, Hedayat T. Female Sex as a Risk Factor for Glycemic Control and Complications in Iranian Patients with Type One Diabetes Mellitus. Iran J Pediatr 21(3):373378(2011)

19. Springer, D, Dziura, J, Tamborlane, WV, et al. Optimal control of type 1 diabetes mellitus in youth receiving intensive treatment. J Pediatr 149(2):227-32(2006) 
20. World Health Organization. WHO Guidelines on physical activity and sedentary behaviour (2020). Geneva: World Health Organization.

21. National Coordinating Committee on Food and Nutrition (2013). Malaysian Dietary Guidelines for Children and Adolescents. Malaysia: Technical Working Group on Nutritional Guidelines.

22. Xiang M, Zhang Z, Kuwahar K. Impact of COVID-19 pandemic on children and adolescents' lifestyle behavior larger than expected. Progress in Cardiovascular Diseases 63(4):531-532(2020). https://doi.org/10/1016/j.pcad.2020.04.013

23. Tornese G, Ceconi V, Monasta L, Carletti C, Faleschini E, Barbi E. Glycemic Control in Type 1 Diabetes Mellitus During COVID-19 Quarantine and the role of In-Home Physical Activity. Diabetes Technology \& Therapeutics 22(6)(2020)

24. Council on communication and media. Children, Adolescents, and the Media. Pediatrics 132;958(2013). https://doi.org/10.1542/peds.2013-2656

25. Margeirdottir HD, et al. Strong Association Between Time Watching Television and Blood Glucose Control in Children and Adolescents With Type 1 Diabetes. Diabetes Care 30:1567-1570(2007)

26. Paruthi S, Brooks LJ, D'Ambrosio C, Hall WA, Kotagal S, Llyold RM, et al. Recommended Amount of Sleep for Pediatric Populations: A Consensus Statement of the American Academy of Sleep Medicine 12:785-786(2016)

27. Jaser SS, Foster NC, Nelson BA, Kittelsrud JM, DiMeglio LA, Quinn M, Willi SM, Simmons JH, for the T1D Exchange Clinic Network. Sleep in children with type 1 diabetes and their parents in the T1D Exchange. Sleep Medicine 39:108-115(2017) 